\title{
Protein-Based Detection Methods for Genetically Modified Crops
}

\author{
Kausar Malik, Haleema Sadia and \\ Muhammad Hamza Basit \\ Additional information is available at the end of the chapter \\ http://dx.doi.org/10.5772/intechopen.75520
}

\begin{abstract}
The generation of genetically modified (GM) crops is rapidly expanding each and every year around the world. The well-being and quality assessment of these harvests are vital issues with respect to buyers' interests. This drove the administrative specialists to execute an arrangement of extremely strict strategies for the endorsement to develop and use GMOs and to produce an interest in scientific techniques equipped for identifying GM crops. The GM crops have been added to the effective fuse of various attributes by presenting transgenes, for example, Bacillus thuringiensis (Bt) insecticidal qualities, in various crop species. GM crops give critical financial, natural, well-being and social advantages to both small and large agriculturists. The detection strategies incorporate either DNA-based or protein-based measures. Different immunoassays or catalyst connected immunosorbent tests are delicate and more affordable; however, they need experienced technicians. A very simple method, that is, immunochromatographic (ICS) test, is set up in the world, which is modest, compact and simple to utilize. The ICS is a semiquantitative method for indicative screening and semi-measurement of new remote proteins presented through hereditary change of plants. The strip is the easiest method for the assessment of several Bt crop plants for insecticidal quality.
\end{abstract}

Keywords: immunochromatography, lateral flow strips, detection assay, genetically modified (GM), trait-specific

\section{Introduction}

The global population is increasing quickly. Experts recommend that food necessities are probably going to rise significantly in the next 20 years. More than 800 million individuals, 
including 33\% of the number of inhabitants in sub-Saharan Africa, are undernourished. More than $90 \%$ of them are enduring long-term malnutrition and micronutrient insufficiency. Genetic modification of crops can possibly take care of these issues. A hereditarily changed life form (GMO) is a life whose genome has been modified by methods of recombinant DNA technology. This innovation adjusts or embeds at least one quality into a life through genetic modification. GMOs hold extraordinary potential to build trim yield, enhance sustenance quality, decrease input costs and enhance creativity. To date, insect protection and herbicide resilient are the primary business attributes utilized as a part of maize, cotton and soybean [1,2]. These qualities are giving monetary advantages to the agrochemical business, seed markets and agriculturists because of improved profitability. They additionally conceivably advantage the land because of a lessening in the utilization of chemicals or a move to the utilization of all the more naturally agreeable chemicals.

The development of GM crops is progressing, more qualities are rising and a bigger number of sections of land are being planted with GM crops. The arrival of GM harvests and items in the business sectors worldwide has expanded the administrative need to screen and check the nearness and the measure of GM crops in yields. The worldwide region of GM crops expanded from 1.7 million hectares in 1996 to 81 million hectares in 2004, with an expanding extent developed by creating nations. More than 8 million ranchers are profiting from this innovation [3]. Around $90 \%$ of the farmers are small agriculturists from developing nations, who increased their earnings from biotech crops significantly.

The administrative need to screen and confirm the nearness and the measure of GM crops has expanded with the development of the GM crops [4]. Effective monitoring of GM crops must be accomplished with the improvement of proper techniques.

GM crops can be distinguished by identifying the transformed hereditary material at the DNA level, the subsequent protein or phenotype. A few expository techniques, for example, strategies in view of the polymerase chain reaction (PCR) for identifying the incorporated DNA, immunological measures for detecting the subsequent protein or utilizing bioassays to recognize the resultant phenotype have been reported. Western blotting, enzyme-linked immunosorbent assay (ELISA) and parallel stream sticks are common protein-based test techniques [5]. A few other diagnostic advances that can give answers for current specialized issues in the GM test examinations are rising. These techniques incorporate mass spectrometry, chromatography, close infrared spectroscopy, miniaturized scale manufactured gadgets and, specifically, DNA chip innovation (microarrays) and mostly immunoassays. Different immunoassays are being used to determine the genetically modified proteins.

The test on a specimen is, for the most part, a screening test that may distinguish a scope of GMOs. This can be trailed by a particular test to recognize the type of GMO in the sample and additionally intended to measure the quantity of a particular GMO. The lion's share of protein recognition strategies depends on immunoassays for discovery and evaluation of new (outside) proteins presented through the genetic modification of plants. Immunoassay depends 
on the reaction between an antigen and a counteracting agent. Protein detection strategies for the GMO testing shift from those that are generally modest and simple to perform to more refined measures requiring costly instrumentation. Protein detection strategies can be utilized to recognize GM attributes in GM crops [6]. GMO testing has turned into a vital and essential piece of food production to ensure compliance with labeling regulations, to confirm IP frameworks and secure customers by approving "non-GMO" item publicizing claims [7].

Binding assays are in widely being used in laboratories for the detection and quantification of proteins in samples. For biological samples such as urine, whole blood, plasma, serum and other biological fluids, assays are often performed in hospitals and clinical laboratories. These binding assays can likewise be performed in natural, horticultural, veterinary, mechanical athletic lawful/criminological settings and furthermore, snappy discovery of irresistible sicknesses in serological testing of people and creatures. The principles involved in such assays are well known by those skilled in the art. Many such devices have been described and are available commercially. Immunological binding assay is the sandwich assay. However, in clinical laboratories, the use of solid phase chromatographic binding assay devices has become commonplace for their relative ease of use, economy, and reproducibility. Typically, these chromatographic assay devices are comprised of a porous chromatographic medium which acts as the matrix for the binding assay. The sample is added directly or indirectly to one end of the medium and is chromatographically transported to a detection reagent with which it reacts to form a labeled product, which is then transported to a test zone containing an immobilized capture reagent such as a capture antibody, in which the presence, absence or quantity of an analyte can be determined.

This depends on immunochromatography and sidelong stream measures. It identifies with immunomeasure dipsticks, and especially to those test gadgets used to lead immunological and serological restricting tests. This new strip test is low in cost, quick, monetary, convenient and less laborious. It can be utilized to detect qualitatively or semiquantitatively the presence of protein of interest samples. The development in techniques for utilizing such dipsticks for the detection of GMOs is increasing.

GMOs hold the awesome potential to increase crop yield, enhance nutrient quality, lessen input costs and enhance creativity. To date, insect and herbicide resistance are the principal business attributes utilized in maize, cotton and soybean. The worldwide area of GM crops expanded 47-fold, from 1.7 million hectares in 1996 to 81 million hectares in 2004, with an expanding extent developed by developing nations $[2,3]$. GMO testing has turned into an indispensable and essential piece of food production to ensure compliance with labeling regulations. Protein-based methods, for example, ELISA and strip tests are viable for natural items yet rely upon the accessibility of business units and are not appropriate for prepared items because of protein degradation [7]. Parallel stream systems are subjective or semiquantitative [4]. Immunoassays have the ability to be broadly executed on a large scale for the recognition of novel proteins in crude food items. Immunoassay advances are perfect for the subjective and quantitative discovery of many sorts of proteins and pathogens in complex systems [5, 8]. Effective testing of GM products must be accomplished with the improvement 
of proper strategies for detection. These strategies are for the most part in view of the study of the novel proteins or DNA.

For approval of a scientific strategy, the testing objective must be characterized and execution qualities must be illustrated. Execution qualities incorporate exactness, extraction proficiency, accuracy, reproducibility, affectability, specificity and strength. The utilization of approved strategies is essential to guarantee acknowledgment of results delivered by diagnostic research facilities [9]. The greater part of protein detection techniques depends on immunoassays. Protein detection techniques can possibly recognize the nearness of a particular GM quality and to give the total measurement of the level of transgene expression. Protein identification strategies are exceedingly reasonable for checking particular GM attributes amid treatment of crude items, gave the protein is communicated in the piece of the plant being tried.

Here are the details of immunoassays being used for the detection of genetically modified proteins.

\section{Immunoassays}

An immunoassay is a biological test that identify and quantify the micro- or macromolecules with the help of antigen or antibody, and the molecule to be detected is called as an analyte. Specific antigens can be stimulated by specific immune responses and as a result of an immune response in the body, antibodies are produced, which are proteins, and they have a sense to find the presence of any foreign antigen in the body. Immunoassays vary in formats. Multiple steps are involved in these assays where reagents are being added and then extra reagents are washed away. Multistep assays are often called heterogeneous immunoassays or separation immunoassays [10]. A few immunoassays can be performed by mixing the samples and reagents and are nonseparation immunoassays or homogeneous immunoassays. The vital component of an immunoassay is an antibody which has a high specificity for the target molecule (antigen), and the area on antigen where antibody attaches is called as an epitope. Standards or calibrators of known concentration are being used to quantify the unknown concentration of analyte. These detections of antigen or antibody take place with the help of labels attached to the antigen or antibody. Many labels are detectable as either they produce a color change in a solution, emit radiations or can be induced to emit light or fluorescence under UV light. The most common used labels for immunoassays are the enzymes.

\subsection{History}

In the 1950s, the first immunoassay was developed by the Solomon Berson Rosalyn Sussman Yalow. In 1977, Yalow received the Nobel Prize for her work and came in the list of second American women who won this award [11, 12]. In the 1960s [13], the immunoassay became more simple with the discovery of chemically linked enzymes to the antibodies, and later in 1983 [14], Professor Anthony Campbell from Cardiff University introduced acridinium ester in immunoassay that used its own light. This immunoassay helped to quantify a wide range of pathogens, proteins and other proteins in blood samples [14]. 


\section{Classification of immunoassays}

1. Competitive homogenous immunoassays

2. Competitive heterogeneous immunoassays

3. One-site noncompetitive immunoassays

4. Two-site noncompetitive immunoassays

\subsection{Competitive, homogeneous immunoassays}

In competitive homogenous immunoassay, there is a competition between labeled and bound analyte (bound to the antibody) with unlabeled and unbound analyte in the sample. As a competition, the unlabeled and unbound analyte displace the labeled and bound analyte and get them attached in place, while the detached labeled analyte then give fluorescence, and this fluorescence is measured, which is proportional to the amount of unlabeled and initial unbound analyte in the sample.

\subsection{Competitive heterogeneous immunoassay}

In heterogeneous assay, there is a competition between bound and labeled analyte (bound to the antibody) with unbound and unlabeled analyte, the difference from competitive homogenous assay is that the labeled unbound/displaced analyte is separated by washing, and the remaining labeled and bound analyte is measured.

\subsection{One-site noncompetitive immunoassays}

In this immunoassay, the amount of unknown analyte in the sample is measured by adding the labeled antibodies. The labeled antibodies get attached with the analyte in the sample, and the extra labeled unbound antibodies are washed away, so, only labeled and bound antibodies are present in the sample, the intensity of fluorescence of these antibodies is measured, which is proportional to the 3.4. amount of unknown analyte in the sample.

\subsection{Two-site noncompetitive immunoassays}

In this immunoassay, there is an antibody present on a site, and the analyte in a sample is added to the antibody get attached, and then second antibody is added which is attached with the label. If the specific analyte is not present in the solution, the second antibody will not attach. Then, the fluorescence of the labeled antibody is measured, which is directly proportional to the amount of analyte in the sample. It is very important to consider that there are washing steps after every reaction, so extra materials are always washed away. The other thing very important is that what type of labels are attached and how the fluorescence/signal is measured. The details of the labels are given as follows: 


\subsubsection{Radioactive isotopes}

To produce a radioimmunoassay (RIA), radioactive isotopes can be added into the immunoassay reagents and the radiations emitted by bound antigen-antibody complex can be determined by the conventional methods. RIA is considered as the earliest developed immunoassay, and they are not used frequently nowadays because of the hazards of radioactivity $[16,17]$.

\subsubsection{Fluorogenic reporters}

Many modern immunoassays are performed by the use of fluorogenic reporters, and the protein microarrays are the best example where these labels are being used [18, 19].

\subsubsection{Electrochemiluminescent tags}

Electrochemical tags are the labels which emit light as a response of electric current, and the chemiluminescence is detected [20].

\subsubsection{DNA reporters}

In real-time quantitative PCR, the traditional immunoassays techniques are added and this is called as real-time immunoquantitative PCR (iqPCR). The labels used in this assay are DNAlabeled probes [21, 22].

\subsubsection{Enzymes}

The most commonly used labels in immunoassays are enzymes, such immunoassays are called as enzyme-linked immunosorbent assays (ELISA) or sometimes enzyme immunoassays (EIAs). Different enzymes are used in such assays, for example, glucose oxidase, horseradish peroxidase (HRM) and alkaline phosphatase. The enzymes are exposed to the reagents which cause them to produce chemiluminescence or light.

\section{Label-free immunoassays}

There are few immunoassays where labels are not required, for example, in one immunoassay, the antigens are measured by change in resistance in the electrode as the antigen attaches to it. In another method, the binding between unlabeled antibody and antigen is detected by resonance and the technique is called as surface plasmon resonance, and these resonance signals are produced by metal nanoparticle tags which can be measured by a microphone $[23,24]$.

Different techniques where the immunoassays are being used are as follows:

1. Radioimmunoassay

2. ELISA 
3. Memory lymphocyte immunostimulation assay (MELISA)

4. Immunoscreening

5. Cloned enzyme donor immunoassay (CEDIA)

6. Lateral flow test

7. Magnetic immunoassay (MIA)

8. Surround, optical fiber immunoassay (SOFIA)

9. Ultra sensitive antibody detection by agglutination-PCR

10. CD/DVD-based immunoassay.

\subsection{Radioimmunoassay}

RIA is an extensive method in which radioactive labels are used in a stepwise manner and as it is very specific and sensitive method which require a special equipment. Another such method is called immunoradiometric assay (IRMA) in which radiolabels are used in an immediate manner rather in steps. Radioallergosorbent test (RAST) is used to determine the allergen in case of allergy. It is the cheapest method to perform immunoassay. Although it is the cheapest method to perform immunoassay, it needs special licensing and precautions as radioactive compounds are being used [25-28].

\subsubsection{Method}

The following steps are required to perform radioimmunoassay:

1. Gamma-radioactive isotopes of iodine, for example, 125-I, attached to the tyrosine are used to label the known amount of antigen

2. A known amount of antibody is mixed with these radiolabelled antigens.

3. Labeled antigen and unlabeled antibody get attached by their binding sites.

4. A sample of serum having the same antigen of unknown amount is added to the mixture.

5. A competition between labeled ("hot") and unlabeled ("cold") antigen is built to attach with antibody binding sites.

6. When the concentration of unknown antigen is increased, it starts to displace the labeled antigen from the antibody binding sites

7. The displaced labeled antigens and bound antigen-antibody complexes got separated, and the radioactivity of displaced radiolabelled antigens is measured by Gamma Counter.

${ }^{*}$ Radioimmunoassay can be performed as same as the sandwich ELISA method (see sandwich ELISA), the difference is that in ELISA, enzyme is linked with secondary antibody, while in this sandwich radioimmunoassay, radioactive compound is used. 


\subsection{Enzyme-linked immunosorbent assay (ELISA)}

ELISA is a type of immunoassay, and the principle behind its working is the same as that of immunoassay, just it is a wet-lab based assay that uses solid phase enzyme and that is why it is also called as enzyme immunoassay (EIA). ELISA is considered as a quality control test in industries and diagnostic tests in hospitals. ELISA falls under the category of ligand binding assays as it involves the binging of antibody and antigen. When labeled antigens or antibodies get attached to substrates, they make a reaction which causes a change in color, and this color is used as a signal. This substrate to enzyme linkage was developed by Stratis Avrameas and G.B. Pierce. As it is very necessary to wash away the unnecessary or unbound chemicals after each reaction, so that is why the bound antigen-antibody complex should be fixed to the surface of the container with the help of immunosorbent, and this method was developed by Jerker Porath and Wide in 1966. In 1971, a group of different scientists Bauke van Weemen and Anton Schuurs, in the Netherlands, and Eva Engvall and Peter Perlmann, in Sweden, independently published papers describing the methods of ELISA/EIA. Usually, chromogenic reporters and substrates are used, which give observable change in color according to the amount of antigen-antibody complex. In ELISA, a solid phase which is physically immobilized is used to absorb certain components of the liquid phase which has the analyte to be detected. Different reagents and solutions are added, incubated and washed off, and in the end, some optical changes take place which are measured by spectrophotometer at specific wavelength. If the antigen is present in the liquid to be diagnosed/detected, then the labeled antibody is added and vice versa, and then, the substrate is added which reacts with the enzyme of labeled antigen or antibody and then stop solution is added to stop the reaction, and the color change is measured at specific wavelengths by ELISA reader. ELISA can give results in two forms [12, 29-31]:

1. Qualitative: In quantitative ELISA, just positive or negative results can be mentioned. A cutoff value is adjusted by running known positive and negative samples, and the optical density of the solution is measured by spectrophotometer.

2. Quantitative: Quantitative ELISA is used for the quantification of analyte, and the series of standards are used and the unknown amount of analyte is measured.

Different kits are also available in the market for each type of ELISA according to the application and requirement. Mostly, the basic principle and methodology are the same. Procedures and reagents are provided with each specific kit along with the methodology.

Following are the four different types of ELISA and their methodologies:

\subsubsection{Direct ELISA}

Direct ELISA comprised of the following steps:

- A liquid solution having analyte to be detected is added to the microtiter plate, one sample per well of the plate. The plate has the solid/plastic phase, which absorbs the analyte by charge exchange. 
- Bovine serum proteins or casein is added to the wells, which are nonreactive in order to cover that portion of plastic which is not covered by antigen.

- Primary antibody having attached enzyme is added, and it binds with the antigen.

- A substrate is added, which changes the color of the solution by reacting with enzymes.

- The higher the concentration of primary antibody in the solution, the higher the color change will be there and higher will be the analyte in the liquid to be tested.

- The major disadvantage of the direct ELISA is that when antigen is to be measured from serum, antigen mobilization become difficult due to many other proteins present in the serum. Sandwich or indirect ELISA becomes more suitable in that case (Figure 1).

\subsubsection{Sandwich ELISA}

Sandwich ELISA is a type of immunosorbent assay in which one antigen is sandwiched between the two antibodies or one antibody is sandwiched between two antigens for more specific reactions. The procedure of the ELISA is given below [32, 33]:

1. A known amount of antibody is bound to a fixed surface.

2. Nonspecific sites on solid surface are blocked by bovine serum albumin, casein or any other such neutral solution.

3. The sample containing antigen is applied to the plate and which is captured by antibody.

4. The unbound antigens are washed away by washing solution

5. The secondary antibody is added which is also labeled with enzymes.

6. The unbound antibodies are washed away.

7. The sandwich is formed having two antibodies and one antigen inside.

8. A substrate is added, and the enzyme reacts with the substrate and gives a color which is proportional to the amount of antigen.

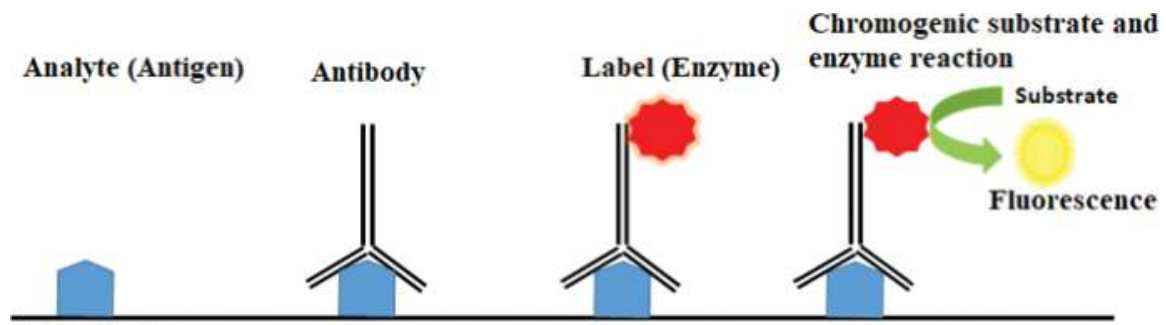

Direct ELISA

Figure 1. Direct ELISA. This figure shows the direct ELISA in which the analyte (antigen) to be determined is attached with the labelled antibody and then chromogenic substrate is added which reacts with the enzyme and gives fluorescence. 
9. The absorbance or fluorescence or electrochemical signal (e.g., current) of samples is measured to determine the presence of antigen and to quantify it (Figure 2).

\subsubsection{Indirect ELISA}

Indirect ELISA is the same as that of the direct ELISA, only primary antibody has been unlabeled, which is very specific to the antigen and the labeled secondary antibody is added, which is labeled withe enzyme or any other label (Figure 3).

\subsubsection{Competitive ELISA}

There is a competition of analyte in this ELISA. The procedure is as follows:

1. First sample having antigen is incubated in the presence of antibody.

2. This antigen-antibody complex is then added into the antigen-coated well.

\section{Seconadry antibody Label with Secondary Antibody}

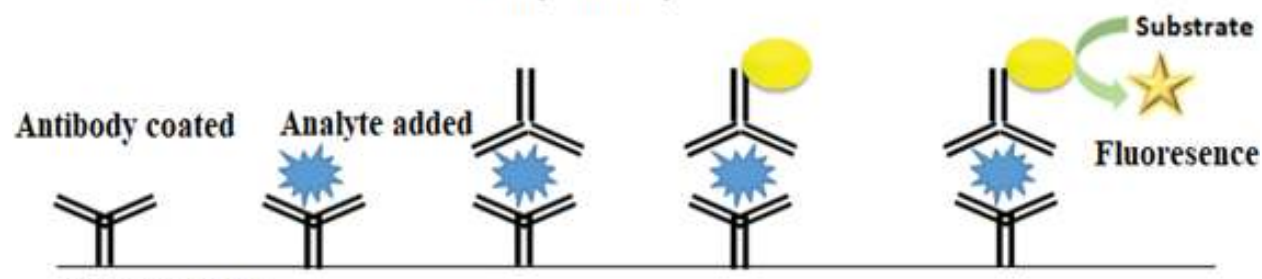

Sandwich ELISA

Figure 2. Sandwich ELISA. This figure shows the antigen is the analyte which is sandwiched between two antibodies, the antibody can be sandwiched between two antigens in the same way.

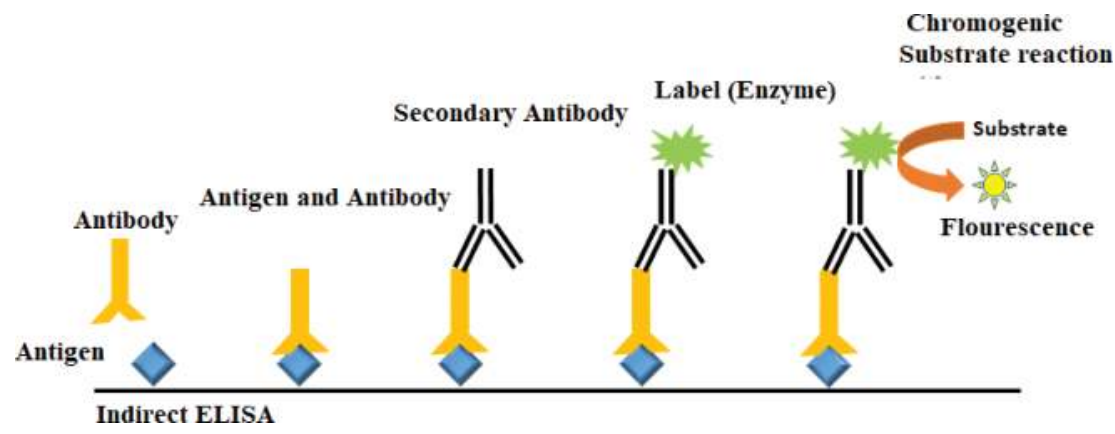

Figure 3. Indirect ELISA. This figure shows the antigen is the analyte to be detected, the primary antibody specific to the antigen is added, secondary antibody is added then, which is labelled with the enzyme and after that chromogenic substrate is added, which reacts with the enzyme and give fluorescence. 
3. The plate is washed to remove all the unbound antibodies, and the antigen-antibody (Ag$\mathrm{Ab}$ ) complex have a competition with labeled antigen as there are less unbound antibodies and coated antigen needs to attach with antibodies which will be taken from bound Ag-Ab complex.

4. Then, the secondary antibody is added which is attached with the enzyme.

5. A substrate is added, and as a reaction of the enzyme and substrate, color is produced.

6. To prevent the eventual saturation of the signal, the stop solution is added to stop the reaction.

In some kits, enzyme-linked antigens are used instead of antibodies, and the remaining competition mechanism is same as described earlier; therefore, there will be a competition of antigens instead of antibodies.

\subsubsection{Applications of ELISA}

To determine the immune response in the body enzyme-linked immunosorbent assay (ELISA) is being used, which have different methods. These methods are being extensively used to determine analyte in the biological samples of whole blood, serum, urine and other biological fluids. These assays have wide applications in agriculture, industrial, environmental, athletic and legal/forensic fields [34-38].

This assay can be used to determine:

1. The antigen present in oncology samples. The elevated levels of carcinoembryonic antigen (CEA) and prostate-specific antigen (PSA) can be used for early diagnosis of tumorigenic processes.

2. Other disorders and diseases can be diagnosed by immunoassays, for example, antigenic determinants of infectious disease organisms, including fungi, viruses and bacteria and yeast. Helicobacter pylori, Mycobacterium tuberculosis, malaria, West Nile Virus, HIV, human papilloma virus (HPV), human chorionic gonadotropin (HCG) for pregnancy, hormones determination, gastrointestinal disorders determination, inherited metabolic diseases determination by enzymes, hexosaminidase as a marker of Tay-Sachs disease and histidase as a marker of histidinemia, tissue damages determination by tissue specific antigen in circulation by determination of creatinine kinase for muscle damage cardiac troponine for myocardial infarction.

3. Suitable identification of the foreign protein in genetically modified organisms (GMOs) and antibodies.

4. To test the athletes' blood sample for recombinant growth hormone, immunoassays are widely used in sports anti-doping laboratories (rGH rhGH, GH, hGH). 


\subsection{Memory lymphocyte immunostimulation assay (MELISA)}

Type-IV hypersensitivity to chemicals, metals and environmental toxins such as molds can be determined by an immunoassay called as a memory lymphocyte immunostimulation assay (MELISA). The test determines the harmful substance in the blood, which is causing allergic reactions, but it will not measure the amounts of toxic substances. Two research articles showed MELISA had many false positive results, while one subsequent study showed that it is very reliable, specific and sensitive method to detect the metals in metal allergic patients [39-43].

\subsection{Immunoscreening}

It is a method to determine the proteins produced by genes inserted into expression vectors. For this, antiserum should be available and the secondary antibody should also be labeled with radioactive compounds or enzymes [44].

\subsection{Cloned enzyme donor immunoassay}

In this type of Immunoassay, in which two types of enzymes are being used which can be active only when they combine together [45]. The one enzyme is conjugated with the same type of specific analyte to be determined and this enzyme complex is called as analyteenzyme-fragment conjugate. The other enzyme attaches to the specific antibody. The analyteenzyme-fragment conjugate is unable to assemble with the other enzyme, if it is attached to the antibody. For this purpose, the antibody should be displaced from the enzyme.

Therefore, when the analyte to be determined present in the serum is mixed with the analyteenzyme-fragment conjugate and antibody-enzyme. There is a competition between the analyte in the serum and the analyte-enzyme-fragment conjugate. If the concentration of analyte is high in the serum, then, it will attach with the antibody-enzyme, and the enzyme will be free to attach with the analyte-enzyme-fragment conjugate to give enzyme activity with the substrate. It means the higher the concentration of analyte in the serum, the higher will be enzyme activity and vice versa.

\subsection{Lateral flow immunochromatographic assays}

Simple devices (Strips) are being used to detect the analyte of interest in the sample without the need of any equipment. A widely used such tests are home pregnancy test, HCV, HBV diagnostic test, and so on. Immunoassays have the ability to be broadly executed on a business scale for the recognition of proteins in food [10, 46-49]. The test is used to detect Bt-GM crops for the expression of insecticidal crystal protein (ICP) of Bacillus thuringiensis. One-step lateral flow tests, which are also called immunochromatographic strips (ICS) or dipstick tests, have been a popular platform for qualitative rapid visual tests, which use colloidal gold conjugate to generate signals.

In previous methodologies, QuickStix lateral flow test devices employ the same immunoassay principles as the plate format, but coat the antibodies and other reagents on a nitrocellulose membrane rather than on the inside of test wells or tubes. Nitrocellulose (NC) membranes have been the first choice of device manufacturers for over 20 years. A test strip assay device, 
in which a mobile conjugate labeled with colloidal labels such as gold, can be deposited on a chromatographic medium, and after reaction with an analyte, thus transported with the solvent to a test zone. The labeled mobilizable detection reagent reacts with an analyte, and the resulting product migrates with the liquid sample as the sample progresses to the test zone. During manufacturing, after the unlabeled binding agent is added to and immobilized in the test zone, the remainder of the test strip material is treated with blocking agents, in order to block any remaining binding sites. The zone where the mobilizable labeled reagent is located is often referred to as the "labeling zone," but can be referred to as the "reversible immobilization zone" or "mobilization zone" while the analyte is reacting with the mobilized labeled reagent, the liquid sample and mobilized labeled reagent migrates further within the porous carrier to the detection zone, where reagent that binds the same analyte is fixed or immobilized, usually in the form of a line. The important aspects of antibody pairs include steric separation of epitopes, an adequate titer of stocks, high affinity, high specificity, high avidity and purity.

The benefits of immunochromatographic tests include user-friendly format, very short time to get a test result, long-term stability over a wide range of climates and relatively inexpensive to make. These features make strip tests ideal for applications such as home testing, rapid point of care testing and testing in the field for various environmental and agricultural analytes. It is limited to diagnostic screening applications only. Furthermore, the achievable sensitivity is a factor of about 10-100 poorer than an instrumented laboratory immunoassay, restricting the technology's utility to relatively high abundance analytes only. Some of the more common lateral flow tests currently on the market are tested for pregnancy, strep throat [50], Chlamydia and human brucellosis [51]. Lateral flow assays have been used extensively as diagnostic tools for monitoring of toxins (Figure 4).

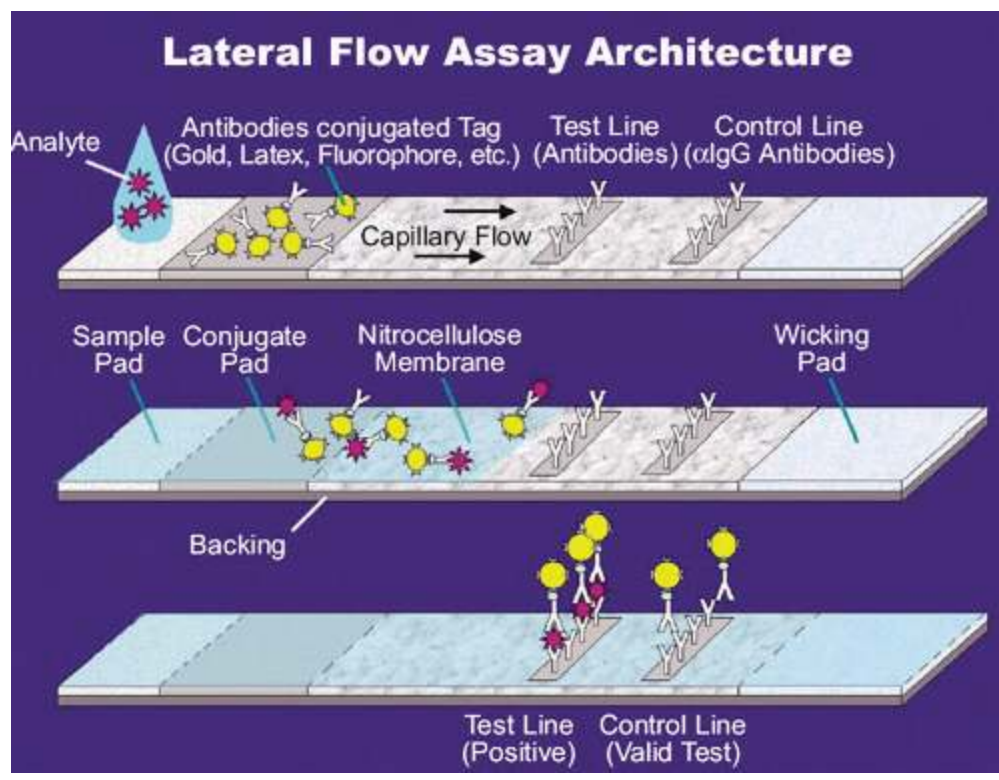

Figure 4. Lateral flow method. 


\subsection{Magnetic immunoassay (MIA)}

The magnetic nanoparticles were discovered by Frenchman Louis Néel, and he got the first Nobel Prize in Physics in 1970. The scientists described the superparamagnetic quality of these magnetic nanoparticles in the magnetic field. These component magnetic nanoparticles are in the range of 5-50 nm while the magnetic beads may be in the range of $35 \mathrm{~nm}-4.5 \mu \mathrm{m}$. A novel type of diagnostic immunoassay was developed by using these magnetic beads as labels. The presence of magnetic labels is measured by the magnetic reader, that is, magnetometer. Therefore, the signals measured by the instrument are directly proportional to the analyte in the serum (toxin, cardiac marker, virus, bacteria). The superparamagnetic quality of these beads has already been in practice in magnetic resonance imaging (MRI) [52].

\subsection{Surround, optical fiber immunoassay (SOFIA)}

A billion times more sensitive and dynamic technique than conventional diagnostic methods is "surround, optical fiber immunoassay (SOFIA)" for in vitro diagnostics, in which surround optic fiber assembly is used to capture the fluorescence from the sample. SOFIA's sensitivity is up to attograms level, that is, $\left(10^{-18} \mathrm{~g}\right)$. SOFIA has a power to differentiate the analyte over 10 orders of magnitude. This technique is used for ante mortem screening test for, Scrapie, BSE, vCJD, CWD, Alzheimer's, Parkinson's disease and transmissible spongiform encephalopathies [53].

\subsection{Ultra sensitive antibody detection by agglutination-PCR (ADAP)}

With this technique, the antibodies in the ultrasensitive solution are detected by synthetic antigen-DNA conjugates, which enable the ligation of strands of DNA, and quantification is done by qPCR. ADAP can detect zepto- to attomoles of antibodies with dynamic range of $5-6$ orders of magnitude in $2 \mu \mathrm{L}$ of the sample. Agglutination-PCR gives 1000-fold increased sensitive results in the determination of the anti-thyroglobulin autoantibodies from human patient plasma. The ADAP is very sensitive, and very cheap equipment such as Slip Chip is being used, and there is no need to use hazardous radioactive compounds [54].

\subsection{CD/DVD-based immunoassay}

Storage and retrieval of information can be performed on the metal reflective layer and the polycarbonate surface of CD/DVD. The metal surface of the CD is made of pure gold sometimes, and it shows perfect optical activities and this metal can perform the activity of the substrate and compounds can attach to it and as a result, it can change the refractive and reflective properties of the disk, and the signals produced can tell the amount of analyte in the sample.

In addition to the abovementioned immunoassays, there are many other ELISA-based immunoassays, the difference is that ELISA is used to determine the analyte in the liquid solution while these methods are being used to determine analyte in the tissue samples after performing a series of steps, provided with easy time of assay, for example, Western blot, immunohistochemistry, dot blot, immunocytometry, immunostaining. It is very important to know that in immunoassays, there is an importance of antibodies of immunoglobulin, but scientists are working hard to make this procedure more cheaper. 


\section{Aptamers}

Aptamers are single-stranded oligonucleotides of DNA or RNA molecules, and have property to bind with high affinity and specificity to their target due to their strong interactions and nanosize, respectively. This property of aptamers can be used for a number of applications in biomedical research, their high efficiency of molecular recognition makes them effective biosensors and therefore, they can be used to develop assays against different targets [55]. Different aptamers can be synthesized for a specific target through a process called systematic evolution of ligands by exponential enrichment (SELEX). Biosensing property of aptamers offers fast and easy detection of target molecules. This property can be used for diagnosis and other biomedical applications, which will help to fight against a number of diseases, including AIDS, cancer, Alzheimer's, viral and bacterial infections. A number of aptamers can be identified against various targets, including nucleotides, proteins, lipids, signaling molecules and even whole cells and microorganisms. Recent advances in research have proven that RNA aptamers have high therapeutic and diagnostic value. It can also be used for therapeutic delivery of oligos. All these attributes of aptamers make them pivotal tools of the emerging bionanotechnology and biosensors. Some research groups are working on aptamer technology and using them as aptasensors but it requires more attention to boost our research for diagnosis and fight against different diseases. Aptamers are easy to synthesize and more stable as compared to antibodies; therefore, they can be helpful in our future advances in therapeutics and diagnosis [56].

\section{Author details}

Kausar Malik $^{1 *}$, Haleema Sadia ${ }^{2}$ and Muhammad Hamza Basit ${ }^{1}$

*Address all correspondence to: kausarbasit786@yahoo.com

1 National Centre of Excellence in Molecular Biology, University of the Punjab, Lahore, Pakistan

2 Department of Biotechnology and Informatics, Balochistan University of Information Technology, Engineering and Mangement Sciences, Lahore, Pakistan

\section{References}

[1] Dunwell JM. Novel food products from genetically modified crop plants: methods and future prospects. International Journal of Food Science and Technology. 1998;33(3): 205-213

[2] James C. Preview: Global status of commercialized transgenic crops: ISAAA briefs No. 30. Ithaca, NY: ISAAA; 2003

[3] Matsuoka T, Kuribara H, Akiyama H, Miura H, Goda Y, James C. Preview: Global Status of Commercialized Biotech/GM Crops; 2004 
[4] Tripathi L. Review: Techniques for detecting genetically modified crops and products. African Journal of Biotechnology. 2005;4(13):1472-1479

[5] Brett GM, Chambers SJ, Huang L, Morgan MRA. Design and development of immunoassays for detection of proteins. Food Control. 1999;10(6):401-406

[6] Stave JW, Magin K, Schimmel H, Lawruk TS, Wehling P, Bridges A. AACC collaborative study of a protein method for detection of genetically modified corn. Cereal Foods World. 2000;45:497-501

[7] Spiegelhalter F, Lauter F-R, Russell JM. Detection of genetically modified food products in a commercial laboratory. Journal of Food Science. 2001;66(5):634-640

[8] Ahmed FE. Detection of genetically modified organisms in foods. Trends in Biotechnology. 2002;20:215-223

[9] Lipp M, Anklam E, Stave JW. Validation of an immunoassay for detection and quantification of genetically modified soybean in food and food fractions using reference materials: Interlab Study. Journal of AOAC International. 2000;83:919-927

[10] Yetisen AK. Paper-based microfluidic point-of-care diagnostic devices. Lab on a Chip. 2013;13(12):2210-2251. DOI: 10.1039/C3LC50169H

[11] Rall JE, Berson SA. In "Biographical Memoirs". National Academy of Sciences. 1990;59:54-71. ISBN: 0-309-04198-8. Fulltext

[12] Yalow RS, Berson SA. Immunoassay of endogenous plasma insulin in man. The Journal of Clinical Investigation. 1960;39:1157-1175. DOI: 10.1172/JCI104130. PMC 441860. PMID: 13846364

[13] Lequin R. Enzyme immunoassay (EIA)/enzyme-linked immunosorbent assay (ELISA). Clinical Chemistry. 2005;51(12):2415-2418. DOI: 10.1373/clinchem.2005.051532. PMID: 16179424

[14] Prof Anthony Campbell - MA PhD. Cardiff University. Retrieved 29 December 2012

[15] NPS Focus. Rainbow Makers. Royal Society of Chemistry (RSC); 2003. Retrieved 29 December 2012

[16] Landers SJ. ELISA test marks 35 years of answering medical questions. American Medical News. 3 April 2006. Retrieved 9 December 2012

[17] Yalow RS. America.gov. April 27, 2008. Retrieved June 26, 2010

[18] Rajkovic E-M. Immunoquantitative real-time PCR for detection and quantification of Staphylococcus aureus enterotoxin B in foods". Applied and Environmental Microbiology. 2006;72(10):6593-6599. DOI: 10.1128/AEM.03068-05. PMC 1610299. PMID: 17021210

[19] Gofflot El. Immuno-quantitative polymerase chain reaction for detection and quantitation of prion protein. Journal of Immunoassay and Immunochemistry. 2004;25(3): 241-258. DOI: 10.1081/ias-200028044. PMID: 15461386 
[20] Luminex xMAP Technology. Millipore Corporation. Retrieved 13 December 2012

[21] Chatterjee S. Protein microarray on-demand: a novel protein microarray system. PLoS ONE. 2008;3:e3265. DOI: 10.1371/journal.pone.0003265. PMC 2533396. PMID: 18813342

[22] Forster RJ, Bertoncello P, Keyes TE. Electrogenerated chemiluminescence. Annual Review of Analytical Chemistry. 2009;2:359-385. DOI: 10.1146/annurev-anchem-060908-155305. PMID: 20636067

[23] González-Díaz JB et al. Plasmonic Au/Co/Au nanosandwiches with enhanced magnetooptical activity. Small. 2008;4(2):202-205. DOI: 10.1002/smll.200700594. PMID: 18196506

[24] Tsekenis G. Label-free immunosensor assay for myelin basic protein based upon an ac impedance protocol. Analytical Chemistry. 2008;80(6):2058-2062. DOI: 10.1021/ac702070e

[25] Vare EA, Ptacek G. Patently Female: From AZT to TV Dinners: Stories of Women Inventors and Their Breakthrough Ideas. New York: Wiley; 2002. p. 99. ISBN: 0471023345

[26] Radioimmunoassay at the US National Library of Medicine Medical Subject Headings $(\mathrm{MeSH})$

[27] http://users.rcn.com/jkimball.ma.ultranet/BiologyPages/R/Radioimmunoassay.html

[28] http://www.biomnis.com/en

[29] Wide L, Porath J. Radioimmunoassay of proteins with the use of Sephadex-coupled antibodies. Biochimica et Biophysica Acta. 1966;130(1):257-260. DOI: 10.1016/0304-4165(66) 90032-8

[30] Engvall E, Perlmann P. Enzyme-linked immunosorbent assay (ELISA) quantitative assay of immunoglobulin G. Immunochemistry. 1971;8(9):871-874. DOI: 10.1016/00192791(71)90454-X. PMID: 5135623

[31] Van Weemen BK, Schuurs AHWM. Immunoassay using antigen-Enzyme conjugates. FEBS Letters. 1971;15(3):232-236. DOI: 10.1016/0014-5793(71)80319-8

[32] Schmidt SD, Mazzella MJ, Nixon RA, Mathews PM. A $\beta$ measurement by enzymelinked immunosorbent assay. Methods in Molecular Biology. 2012;849:507-527. DOI: 10.1007/978-1-61779-551-0_34. PMID: 22528112

[33] Kragstrup TW, Vorup-Jensen T, Deleuran B, Hvid M. A simple set of validation steps identifies and removes false results in a sandwich enzyme-linked immunosorbent assay caused by anti-animal IgG antibodies in plasma from arthritis patients. 2013

[34] MedlinePlus Encyclopedia ELISA/Western blot tests for HIV

[35] Food Allergen Partnership" (Press release). FDA. January 2001. Retrieved August 20, 2015

[36] Sblattero D, Berti I, Trevisiol C, Marzari R, Tommasini A, Bradbury A, Fasano A, Ventura A, Not T. Human recombinant tissue transglutaminase ELISA: An innovative diagnostic assay for celiac disease. The American Journal of Gastroenterology. 2000;95(5): 1253-1257. DOI: 10.1111/j.1572-0241.2000.02018.x. PMID: 10811336 
[37] Porcelli B, Ferretti F, Vindigni C, Terzuoli L. Assessment of a test for the screening and diagnosis of celiac disease. Journal of Clinical Laboratory Analysis. 2016 Jan;30(1):65-70. DOI: $10.1002 /$ jcla.21816

[38] Griffin JFT, Spittle E, Rodgers CR, Liggett S, Cooper M, Bakker D, Bannantine JP. Immunoglobulin G1 enzyme-linked immunosorbent assay for diagnosis of Johne's disease in red deer (Cervus elaphus). Clinical and Vaccine Immunology. 2005;12(12):1401-1409. DOI: 10.1128/CDLI.12.12.1401-1409.2005. PMC 1317074. PMID: 16339063

[39] Cederbrant K, Gunnarsson LG, Hultman P, Norda R, Tibbling-Grahn L. In vitro lymphoproliferative assays with $\mathrm{HgCl}_{2}$ cannot identify patients with systemic symptoms attributed to dental amalgam. Journal of Dental Research. Aug 1999;78(8):1450-1458. DOI: 10.1177/00220345990780081101. PMID: 10439033

[40] Cederbrant K, Hultman P, Marcusson JA, Tibbling L. In vitro lymphocyte proliferation as compared to patch test using gold, palladium and nickel. International Archives of Allergy and Immunology. Mar 1997;112(3):212-217. DOI: 10.1159/000237456. PMID: 9066505

[41] Valentine-Thon E, Schiawara HW. Validity of MELISA for metal sensitivity testing. Neuroendocrinology Letters. February-April 2003;24(1-2):57-64. PMID: 12743534

[42] Stejskal VD, Forsbeck M, Nilsson R. Lymphocyte transformation test for diagnosis of isothiazolinone allergy in man. Journal of Investigative Dermatology. June 1990;94(6):798802. DOI: 10.1111/1523-1747.ep12874656. PMID: 1693940

[43] Willis CM, Young E, Brandon DR, Wilkinson JD. Immunopathological and ultrastructural findings in human allergic and irritant contact dermatitis. British Journal of Dermatology. September 1986;115(3):35-16. DOI: 10.1111/j.1365-2133.1986.tb05745.x. PMID: 3530310

[44] Karam J. Methods in Nucleic Acids Research. Boca Raton: CRC Press; November 26, 1990. p. 309. ISBN: 0849353114

[45] Burtis CA, editor. Tietz Textbook of Clinical Biochemistry and Molecular Diagnostics. USA: Elsevier Saunders; 2012. p. 393. ISBN: 9781416061649

[46] Concurrent Engineering for Lateral-Flow Diagnostics (IVDT archive, Nov 99). Archived 2014-04-15 at the Wayback Machine

[47] "Archived copy". Archived from the original on 2012-07-28. Retrieved 2012-07-27

[48] Hansson J, Yasuga H, Haraldsson T, van der Wijngaart W. Synthetic microfluidic paper: High surface area and high porosity polymer micropillar arrays. Lab on a Chip. 2016;16:298-304. DOI: 10.1039/C5LC01318F

[49] Guo W, Hansson J, van der Wijngaart W. Viscosity Independent Paper Microfluidic Imbibition" (PDF). MicroTAS 2016; Dublin, Ireland; 2016

[50] Edwards EA, Phillips IA, Sulter WC. Diagnosis of group A streptococcal infections directly from throat secretions. Journal of Clinical Microbiology. 1982;15:481-483 
[51] Smits HL, Abdoel TH, Solera J, Clavijo E, Diaz R. Immunochromatographic Brucella specific immunoglobulin $\mathrm{M}$ and $\mathrm{G}$ lateral flow assays for rapid serodiagnosis of human brucellosis. Clinical and Diagnostic Laboratory Immunology. 2003;10(6):1141-1146

[52] Nikitin PI, Vetoshko PM, Ksenevich TI. Magnetic immunoassays. Sensor Letters. 2007;5: $1-4$

[53] Rubenstein R et al. Prion disease detection, PMCA kinetics, and IgG in urine from sheep naturally/experimentally infected with scrapie and deer with preclinical/clinical chronic wasting disease. Journal of Virology. September 2011;85(17):9031-9038. DOI: 10.1128/ jvi.05111-11. PMC 3165845. PMID: 21715495. Retrieved 2011-08-21

[54] Tsai C-T, Robinson PV, Spencer CA, Bertozzi CR. Ultrasensitive antibody detection by agglutination-PCR (ADAP). ACS Central Science. 2016;2(3):139-114

[55] Morais S, Puchades R, Maquieira Á. Disc-based microarrays: Principles and analytical applications. Analytical and Bioanalytical Chemistry. July 2016;408(17):4523-4534. DOI: 10.1007/s00216-016-9423-1. PMID: 26922341

[56] Mills DR, Peterson RL, Spiegelman S. An extracellular Darwinian experiment with a self-duplicating nucleic acid molecule. Proceedings of the National Academy of Sciences of the United States of America. July 1967;58(1):217-224. DOI: 10.1073/pnas.58.1.217. PMC 335620. PMID: 5231602 
